2 Research Square
Preprints are preliminary reports that have not undergone peer review.

They should not be considered conclusive, used to inform clinical practice, or referenced by the media as validated information.

\title{
The Feasibility and Utility of Hair Follicle Sampling To Measure FMRP and FMR1 mRNA in Children With or Without Fragile X Syndrome
}

\author{
Jean A. Frazier \\ University of Massachusetts Medical School \\ Mark Roth \\ Fulcrum Therapeutics \\ David M. Cochran \\ University of Massachusetts Medical School \\ Ann Foley \\ University of Massachusetts Medical School \\ Taylor Merk \\ University of Massachusetts Medical School \\ Lauren Venuti \\ University of Massachusetts Medical School \\ Lucienne Ronco \\ Fulcrum Therapeutics \\ Shane Raines \\ Fulcrum Therapeutics \\ Diego Cadavid \\ Fulcrum Therapeutics
}

Isha Jalnapurkar ( $\square$ Isha.Jalnapurkar@umassmemorial.org )

University of Massachusetts Medical School https://orcid.org/0000-0003-2437-9898

\section{Research}

Keywords: Fragile X, FMR1, FMRP, hair follicle, clinical biomarker

Posted Date: December 15th, 2021

DOI: https://doi.org/10.21203/rs.3.rs-1128569/v1

License: @ (i) This work is licensed under a Creative Commons Attribution 4.0 International License. Read Full License 


\section{Abstract \\ Background:}

Fragile X syndrome (FXS) is the most common cause inherited cause of intellectual disability in males and the most common single gene cause of autism. This X-linked disorder is caused by an expansion of a trinucleotide CGG repeat (>200 base pairs) on the promotor region of the fragile X mental retardation 1 gene (FMR1). This leads to the deficiency or absence of the encoded protein, Fragile X mental retardation protein (FMRP). FMRP has a central role in the translation of mRNAs involved in synaptic connections and plasticity. Recent studies have demonstrated the benefit of therapeutics focused on reactivation of the FMR1 locus towards improving key clinical phenotypes via restoration of FMRP and ultimately disease modification. A key step in future studies directed towards this effort is the establishment of proof of concept (POC) for FMRP reactivation in individuals with FXS. For this it is key to determine the feasibility of repeated collection of tissues or fluids to measure FMR1 and FMRP.

\section{Methods:}

Individuals, ages 3 to 22 years of age, with FXS and those who were typically developing participated in this single-site pilot clinical biomarker study. The repeated collection of hair follicles was compared with the collection of blood and buccal swabs for detection of FMR1 mRNA and FMRP and related molecules.

\section{Results:}

There were $n=15$ participants, of whom 10 had a diagnosis of FXS ( $7.0 \pm 3.56$ years) and 5 were typically developing (8.2 \pm 2.77 years). Absolute levels of FMRP and FMR1 mRNA were substantially higher in healthy participants compared to full mutation and mosaic FXS participants, and lowest in the FXS boys. Measurement of FMR1 and FMRP levels by any method did not show any notable variation by collection location at home versus office across the various sample collection methodologies of hair follicle, blood sample, and buccal swab.

\section{Conclusion:}

Findings demonstrated that repeated sampling of hair follicles in individuals with FXS, in both, home and office settings, is feasible, repeatable, and can be used for measurement of FMR1 and FMRP in longitudinal studies.

\section{Introduction:}

Fragile X syndrome (FXS) is an X-linked genetic condition associated with an expansion of the trinucleotide (cytosine-guanine-guanine) CGG repeat within the $5^{\prime}$ untranslated region of the fragile $\mathrm{X}$ mental retardation 1 (FMR1) gene (1). The majority of cases are caused by epigenetic silencing resulting from expanded CGG repeats in intron 1 of the FMR1 gene on the X chromosome that results in hypermethylation of the promoter, heterochromatin formation, and prevention of gene transcription. This results in a deficit of the FMR1-encoded protein fragile X mental retardation protein (FMRP), an RNA-binding protein that regulates dendritic translation and plays a critical role in synaptic development and function (2). FXS can also be caused by mosaicism of transcriptional silencing of the gene, occurring in some but not all of the cells due to either varying size of the repeat expansion or variations in methylation patterns. Mosaicism can result in variability in the production of FMRP (3).

FXS is the most common inherited cause of intellectual disability with a prevalence of 1 in $4000-7000$ for males and 1 in $8000-11,000$ for females (4). Boys are generally more severely affected because the presence in all girls of a second healthy $X$ chromosome with random inactivation of either the healthy or mutated $X$ chromosome in each cell determining the clinical phenotype (5). Variability in methylation and instability of the repeat expansion also contributes to phenotypic heterogeneity. FMRP is expressed in various tissues in the central nervous system (CNS) and periphery and is responsible for several functions including neurogenesis, synaptic plasticity, ovarian functions as well as neuropsychiatric symptoms (6). Clinical manifestations are diverse and vary from mild to severe intellectual disability with variable behavioral impairments which may be related to the level of FMRP (7-9). The most notable clinical phenotypes are deficits in expressive language development and impairment of social interactions with one or more of the following: social anxiety, hyperactivity, and sensory hypersensitivity (10). Autism spectrum disorder (ASD) is a frequent comorbid condition seen in 30 to $43 \%$ of males with FXS and 16 to $20 \%$ of females with FXS (11). FXS is the most common known single-gene cause of ASD (12).

This wide array of cognitive, emotional, and systemic challenges can have significant effects on the academic and daily functioning of individuals with FXS. The majority of the current symptom-based pharmacological treatments are based on FDA-approved treatments for these conditions in the general population or those with other neurodevelopmental disorders $(11,13)$. Advances in experimental models of FXS and other neurodevelopmental disorders with known genetic origins have paved the way for the potential development of disease- and neurobiologic mechanism-specific pharmacological treatments (14). These include novel treatments that target the core deficits at the cellular level, including immature synaptic connections, altered synaptic plasticity, and impaired memory formation, that occur due to lack of FMRP (15).

An innovative approach to therapeutic development in FXS involves directly targeting the proximal event in disease pathogenesis-the transcriptional silencing of the FMR1 gene (16). A limited number of studies utilizing pharmacological approaches to reactivate the FMR1 locus have met with success (17, 18). An important step in enabling testing of potential treatments for the root cause of FXS is development of translational methods to measure reactivation of FMR1 and FMRP in proof of concept (POC). For this it is critical to be able to collect tissues and/or fluids that can be sampled repeatedly and used to measure 
changes in mRNA and protein. Pioneer studies by Willemsen et al (19) showed that hair follicle removal by plucking is feasible and well-tolerated in children with FXS, and that FMRP is readily detectable by immunohistochemistry (IHC) in hair follicles of unaffected relatives and less affected patients. Importantly, they reported that the presence of FMRP in hair follicles was more predictive of intellectual disability (as measured by IQ testing) than FMRP detection in blood. In a recent study, we found a $0.59 \%$ correlation between FMRP measured in isolated peripheral blood mononuclear cells (PBMCs) and intellectual disability in males ages 3-74 years ranging from normal to full mutation (20). However, there is little experience with the feasibility of serial collection of hair follicles by plucking in the clinic (e.g., the work by Prof. Willemsen was done at home). Additionally, optimal (sensitive, specific, and reliable) methods for the measurement of FMR1, FMRP and related molecules in hair follicles is only recently being developed by us (20) and others (21). Keeping these issues in mind, the current pilot study was designed to determine the feasibility of repeated collection of hair follicles in children with FXS for quantitative measurement of FMR1 mRNA and FMRP.

The following questions were investigated by this pilot study: (1) Is repeated sampling of hair follicles by plucking feasible in children with or without Fragile $X$ syndrome at home and in the clinic? (2) Is the measurement of the presence and amount of FMRP by Meso Scale Discovery Enzyme-Linked Immunosorbent Assay (MSD ELISA) less variable and more reliable than with IHC? (3) Are hair follicles a more feasible choice for repeated sampling of tissue/fluid from children with FXS than blood collection from peripheral veins and/or buccal swabs? (4) Which of the fluids/tissues tested for FMR1 mRNA and FMRP better reflect cognitive function, as measured by tests of oral expression and listening comprehension?

\section{Methods:}

This was a small, single center (University of Massachusetts Chan Medical School (UMMS)), prospective, non-drug pilot feasibility study of the repeated collection of hair follicles by plucking in individuals, 3 to 22 years of age, with FXS and those who were typically developing for the quantitative measurement of FMR1 mRNA and FMRP. The repeated collection of hair follicles was compared with the collection of blood and buccal swabs for detection of FMR1 mRNA and FMRP and related molecules in children and young adults with FXS and age and sex-matched children and young adults without FXS (healthy controls). For this study, most participants completed three study visits: Screening Visit, Visit 1, and Visit 2. An unscheduled visit was allowed for additional sample collection, should any of the samples be inadequate for analysis, or if there was a reason the participant could not provide one of the samples at the regularly scheduled visits. If requested by the family and deemed appropriate by the Principal Investigator (PI), Screening Visit and Visit 1 could be combined. There were two sample collection visits, one in the child's home and one at the clinic. The order of the location of the visits could vary (home visit first, office visit second or office visit first, home visit second), depending on the preference of the family. The feasibility for the repeated collection was determined by the two separate visits, 1-59 days apart. There could be one additional unscheduled visit, either in the office or at home, in case of missed or incomplete scheduled visits.

Measurement of FMRP in hair follicles and peripheral blood lymphocytes (PBLs) was performed by MSD ELISA on whole protein extracts. For PBLs, an independent, flow cytometric method was used to simultaneously measure FMR1 and FMRP in addition to the MSD ELISA (20). Measurement of FMR1 mRNA in hair follicles, blood lymphocytes, and buccal swabs was performed by TaqMan analysis.

\section{Participants:}

Individuals 3 to 22 years of age were recruited for this study. Some participants were invited to participate from the FXS clinic at UMMS while other participants were recruited from the greater New England area via referrals from patient organizations and other methods. A parent focus group was formed as part of recruitment efforts for education on the rationale for the study and to gain parent support. Recruitment materials prepared by UMMS were used to facilitate awareness and communication between interested families at the Center for Autism and Neurodevelopmental Disorders (CANDO) clinic at UMMS and University of Massachusetts Memorial Health Care. Informed consent was obtained from the parent/legally authorized representative (LAR) for children ages 3-17 years, by self (ages 18-22) or by proxy (parent or LAR of the individual). If they were capable, assent by the children of their willingness to have samples of hair, blood and buccal swabs collected was obtained.

The determination of the FXS phenotype regarding expressive language delay and/or social anxiety was performed by the principal investigator (JF) based on the history and physical examination, review of available medical records, and results of standardized testing. All subjects were assessed at screening with standard and age-appropriate tests of language development, social anxiety, social communication impairment, and other FXS phenotypes. The OWLS-II Listening Comprehension (LC) and Oral Expression (OE) portions were used to measure expressive language and LC (22). The Gilliam Autism Rating Scale 3rd Edition (GARS-3) was used for the determination of the presence and severity of restrictive/repetitive behaviors, social interaction impairment, social communication impairment, altered emotional responses, cognitive style, and maladaptive speech by parent report (23).

\section{Procedures:}

\section{Hair Follicle Collection and Analysis:}

Plucking refers to the process of removing human hair by mechanically pulling hair follicles from the scalp. In this study, hair follicles were collected via plucking at Visit 1, Visit 2, and any Unscheduled Visits, using methodology and tweezers provided by Epistem Ltd. Epistem and Fulcrum provided training to UMMS personnel via Webex with ongoing support as needed, including on the collection and the quality assessment of plucked hairs. Required supplies were provided for hair follicle storage. It was anticipated that about 20 hair follicles would be plucked per visit, of which about 15 would pass quality control. Any plucked hairs missing the follicle were discarded by Epistem or Fulcrum.

\section{Collection and Analysis of Blood Cells, Cellular DNA and Plasma:}


Blood was collected via venipuncture at Visit 1, Visit 2, and any Unscheduled Visits. At Visit 1, blood was collected for both FMR1 methylation assessment and FMR1/FMRP measurement. The blood collection at Visit 2 or any Unscheduled Visit was optional for subjects with FXS.

Measurement of FMRP in hair follicles was performed at Fulcrum by MSD ELISA on whole protein extracts from individual hair follicles. Measurement of mRNA in hair follicles was performed by TaqMan analysis. The blood collection tubes were shipped to Fulcrum where peripheral blood lymphocytes, DNA, and serum isolation was performed. Claritas Genomics also performed the analysis for the FMR1 gene mutation for mosaicism and length of the triple repeat expansion when not available from the clinical records. Blood lymphocytes and plasma were evaluated for the measurement of FMR1 mRNA or FMRP. FACS analysis was performed at Fulcrum. For complete MSD ELISA and FACS methods, refer to Roth et al. 2021.

Measurement of FMRP by IHC was discontinued early due to fragility of youth hair follicles (Epistem Ltd., UK). Measurement of FMRP in serum using MSD ELISA was performed at Fulcrum or by NanoSomiX by isolation and characterization of exosomes. Buccal swabs were obtained using the manufacturer's instructions (Puritan HydraFlock from Puritan Diagnostics [Guilford, ME]) at Visit 1, Visit 2, and any Unscheduled Visits. Measurement of FMRP was performed by MSD at Fulcrum by MSD ELISA of protein extracts. Measurement of FMR1 mRNA in buccal swabs was performed at Fulcrum by TaqMan analysis. Measurement of FMR1 by qRT-PCR was not done in PBMCs due to lack of sufficient sample to run both, protein assays and qRT-PCR. We were able to obtain FMR1 expression by PrimeFlow ${ }^{\mathrm{TM}}$ because the detection of FMR1 and FMRP were done on the same sample.

All hair follicles, blood tubes, and buccal swabs were shipped by the primary study site on the same day as the collection under conditions specified in the study reference manual. All samples were labeled with study identification number and fully anonymized prior to shipping. Fulcrum scientists shipped a portion of the hair follicles (about 5 per subject per visit) to Epistem for analysis by IHC.

\section{Statistical Analysis:}

Data analysis was performed using statistical analysis software (SAS) Version 9.4. Descriptive statistics for continuous variables included number of subjects (n), mean, standard deviation (SD), median, minimum, and maximum. Summaries of change from baseline variables included only subjects who had both a baseline value and corresponding value at the timepoint of interest. Descriptive statistics for categorical data included frequency and percentage. Where appropriate, descriptive statistics were presented with 95\% confidence intervals (Cls). Measures of spread (e.g., standard deviation) were reported to 2 degrees of precision more than the recorded data. Pearson's correlations were used to assess relationships between FMR1 and FMRP levels with measures of OWLS-II LC and LE portions and the GARS-3 subscales.

\section{Results:}

\section{Demographic and Other Baseline Characteristics:}

Overall, 15 participants were enrolled in the study, with 10 participants in the FXS participant group and 5 participants in the healthy participants group. All enrolling participants (100\%) completed the study, and none discontinued from the study. There were no screen failures.

The overall demographic and baseline characteristics of subjects in the All Enrolled Set are presented in Table 1. Educational indicators differed between the two groups. The mean age of FXS participants was $7.0 \pm 3.56$ years. A majority of FXS participants $(60.0 \%)$ were male versus $40.0 \%$ female. The mean age of healthy participants was $8.2 \pm 2.77$ years. A majority of healthy participants $(60.0 \%)$ were female versus $40.0 \%$ male. The enrolled population was predominantly Caucasian (93.3\%) and Not Hispanic or Latino (93.3\%). 


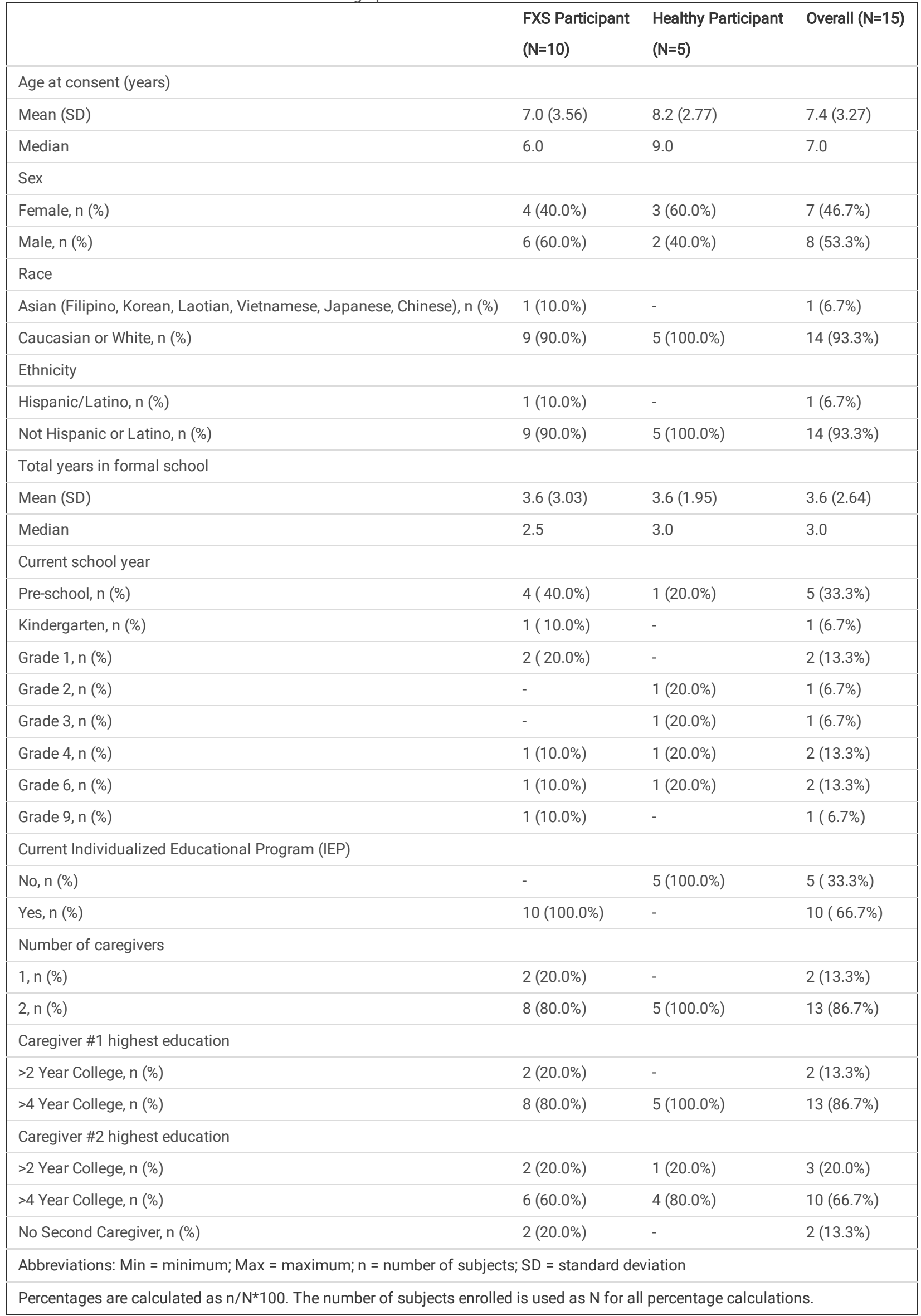


Overall, the majority of FXS participants (70.0\%) were currently attending either pre-school (40.0\%), kindergarten (10.0\%), or Grade 1 (20.0\%) while $80 \%$ of healthy participants currently attended from Grade 2 through Grade 6 . The total number of years in formal school was $3.6 \pm 3.03$ for FXS participants and 3.6 \pm 1.05 for healthy participants. All FXS participants (100\%) and no healthy participants (0\%) reported participation in Individualized Educational Programs.

A majority of FXS participants ( $80 \%$ ) had 2 caregivers. A majority of FXS participants had caregivers with 4 years of college or more as their highest education level ( $80 \%$ for caregiver \#1 and $60 \%$ for caregiver \#2). All healthy participants (100\%) had 2 caregivers of which all except 1 caregiver had 4 years of college or more as their highest education level.

Hair samples were collected at each visit. Blood collection for FMR1 methylation was only collected in 6 of 10 FXS participants (1/4 collected at home and $5 / 6$ collected at office).

Baseline disease characteristics for the FXS participants are presented in Table 2. All FXS participants had their prior FXS diagnosis documented by genetic testing. Four FXS participants (40\%) had mosaic positive disease. On both the OWLS-II LC and OE scales, participants demonstrated low oral receptive and expressive communication function at baseline; the mean score was $82 \pm 16.7$ for OWLS-II LC and $73 \pm 16.8$ for OWLS-II OE. 


\begin{tabular}{|c|c|}
\hline & $\begin{array}{l}\text { FXS Participant } \\
(\mathrm{N}=10)\end{array}$ \\
\hline \multicolumn{2}{|c|}{ Genetic confirmation of FXS } \\
\hline Yes, n (\%) & $10(100.0 \%)$ \\
\hline \multicolumn{2}{|c|}{ Mosaic positive } \\
\hline No, n (\%) & $6(60.0 \%)$ \\
\hline Yes, n (\%) & $4(40.0 \%)$ \\
\hline \multicolumn{2}{|c|}{ Tissue or fluid tested } \\
\hline Blood, n (\%) & $10(100.0 \%)$ \\
\hline \multicolumn{2}{|c|}{ OWLS-II Listening Comprehension standard score } \\
\hline Mean (SD) & $82.0(16.69)$ \\
\hline Median & 84.0 \\
\hline \multicolumn{2}{|c|}{ OWLS-II Oral Expression standard score } \\
\hline Mean (SD) & $73.0(16.78)$ \\
\hline Median & 78.0 \\
\hline \multicolumn{2}{|c|}{ GARS-3 Social Communication scaled score } \\
\hline Mean (SD) & $6.7(3.06)$ \\
\hline Median & 7.0 \\
\hline \multicolumn{2}{|c|}{ GARS-3 Emotional Responses scaled score } \\
\hline Mean (SD) & $7.7(2.95)$ \\
\hline Median & 7.0 \\
\hline \multicolumn{2}{|c|}{ GARS-3 Restricted/Repetitive Behaviors scaled score } \\
\hline Mean (SD) & $7.7(3.13)$ \\
\hline Median & 7.0 \\
\hline \multicolumn{2}{|c|}{ GARS-3 Cognitive Style scaled score } \\
\hline Mean (SD) & $8.0(2.18)^{*}$ \\
\hline Median & 8.0 \\
\hline \multicolumn{2}{|c|}{ GARS-3 Social Interaction scaled score } \\
\hline Mean (SD) & $5.3(2.26)$ \\
\hline Median & 4.5 \\
\hline \multicolumn{2}{|c|}{ GARS-3 Maladaptive Speech scaled score } \\
\hline Mean (SD) & $7.8(2.05)^{\star}$ \\
\hline Median & 8.0 \\
\hline \multicolumn{2}{|c|}{ Percentages are calculated as $\mathrm{n} / \mathrm{N}^{\star} 100$. The number of subjects enrolled is used as $\mathrm{N}$ for all percentage calculations. } \\
\hline *N=9 & \\
\hline
\end{tabular}

\section{FMR1 and FMRP in hair follicles:}

A similar number of hair follicles were obtained using repeated collection at the home and office locations in both the FXS and healthy participant groups. For the FXS group, a mean of $4.7 \pm 1.00$ (median 5.0) hair follicles were collected per subject for the 9 subjects who completed the office visit. The 10th subject provided 7 follicles; however, the data was unreliable and not reported. A mean of $6.1 \pm 2.23$ (median 5.5) hair follicles per subject were collected for the 8 FXS subjects at the home visit. For the 5 healthy controls, a mean of $5.2 \pm 2.17$ (median 4.0) hair follicles were collected per subject at the office visit compared to a mean of $4.8 \pm 1.79$ (median 4.0 ) hair follicles per subject at the home visit. 
The majority of the hair follicles obtained from FXS participants were positive above the lower limit of quantification for FMRP using MSD ELISA (office: $81.3 \% \pm 19.65$; home: $59.3 \% \pm 30.35$ ). Similar percentages were seen for the healthy participants in the study (office: $64.1 \% \pm 23.77$; home $70.0 \% \pm 20.92$ ).

Measurement of FMR1 and FMRP levels by any method did not show any notable variation by collection location at home versus office across the various sample collection methodologies (see Table 3). No variation in FMR1 measured by Quantitative reverse transcription polymerase chain reaction (qRT-PCR) was seen for buccal swab samples collected at office versus home locations $(n=7)$ in the FXS population. Values from qRT-PCR and PrimeFlow ${ }^{\text {TM }}$ are not directly comparable. For healthy participants, no variation in FMRP or FMR1 levels was seen for samples collected at office versus home locations. FMRP levels measured by MSD ELISA were similar in hair follicles versus peripheral blood lymphocytes within each group - FXS and healthy participants. However, FMRP levels measured in either hair follicles or PBMCs were much lower in FXS participants compared to healthy participants (see Table 4). In FXS participants, mean FMRP levels in hair follicles at Visit 1 and Visit 2 were $20.7 \pm 23.0 \mathrm{fmol} / \mu \mathrm{g}$ protein and $18.0 \pm 23.7 \mathrm{fmol} / \mu \mathrm{g} \mathrm{protein}$, respectively, compared to $27.3 \pm 31.3 \mathrm{fmol} / \mu \mathrm{g}$ protein and $24.1 \pm 41.8 \mathrm{fmol} / \mu \mathrm{g}$ protein at Visit 1 and Visit 2, respectively, in peripheral blood lymphocytes. FMRP values for healthy participants also did not show any notable difference between the hair follicle (114.5 \pm 21.1 for Visit 1, 109.3 \pm 29.1 for Visit 2) and blood lymphocyte (103.2 \pm 18.0 for Visit 1 and $106.3 \pm 24.0$ for Visit 2) by MSD.

Table 3

FMRP Levels by Collection Type and Collection Location - Descriptive Statistics

\begin{tabular}{|c|c|c|c|c|c|}
\hline Collection Type & Collection Location & $\mathbf{N}$ & Mean & SD & Median \\
\hline \multirow[t]{3}{*}{ Hair Follicle } & FXS Participant ( $\mathrm{N}=9$ ) & 6 & 15.344 & 25.0476 & 4.295 \\
\hline & Office & & & & \\
\hline & Home & 3 & 15.243 & 31.4584 & 1.447 \\
\hline \multirow[t]{2}{*}{ Blood } & Office & 6 & 37.699 & 61.4383 & 1.795 \\
\hline & Home & 1 & 19.681 & 38.1340 & 1.371 \\
\hline \multirow[t]{3}{*}{ Hair Follicle } & Healthy Participant $(\mathrm{N}=5)$ & 5 & 33.746 & 49.3637 & 5.446 \\
\hline & Office & & & & \\
\hline & Home & 5 & 35.464 & 48.3604 & 10.656 \\
\hline \multirow[t]{2}{*}{ Blood } & Office & 5 & 68.415 & 76.7062 & 33.228 \\
\hline & Home & 4 & 63.016 & 73.5248 & 29.732 \\
\hline \multicolumn{6}{|c|}{ Abbreviations: Max = maximum; Min = minimum; $\mathrm{n}=$ number of subjects; $\mathrm{SD}=$ standard deviation } \\
\hline \multicolumn{6}{|c|}{ Notes: For the hair follicle type, the mean fmol FMRP/ug protein value across all samples for each subject is summarized. } \\
\hline
\end{tabular}


Table 4

FMR1 Levels by Collection Type and Collection Location - Descriptive Statistics

\begin{tabular}{|c|c|c|c|c|c|}
\hline Collection Type & Collection Location & $\mathbf{n}$ & Mean & SD & Median \\
\hline \multirow[t]{3}{*}{ Buccal Swab } & FXS Participant $(\mathrm{N}=9)$ & 7 & 0.354 & 0.3782 & 0.363 \\
\hline & Office & & & & \\
\hline & Home & 7 & 0.510 & 0.5799 & 0.247 \\
\hline \multirow[t]{2}{*}{ Hair Follicle } & Office & 9 & 0.090 & 0.1132 & 0.045 \\
\hline & Home & 7 & 0.077 & 0.0904 & 0.040 \\
\hline \multirow[t]{2}{*}{ Blood } & Office & 8 & 1.012 & 0.1052 & 0.990 \\
\hline & Home & 2 & 0.897 & 0.0957 & 0.897 \\
\hline \multirow[t]{3}{*}{ Buccal Swab } & Healthy Participant $(\mathrm{N}=5)$ & 5 & 1.108 & 0.8453 & 0.784 \\
\hline & Office & & & & \\
\hline & Home & 5 & 0.772 & 0.3437 & 0.733 \\
\hline \multirow[t]{2}{*}{ Hair Follicle } & Office & 5 & 0.165 & 0.154 & 0.195 \\
\hline & Home & 5 & 0.184 & 0.1407 & 0.158 \\
\hline \multirow[t]{2}{*}{ Blood } & Office & 4 & 1.197 & 0.0842 & 1.181 \\
\hline & Home & 4 & 1.164 & 0.0604 & 1.152 \\
\hline
\end{tabular}

Abbreviations: Max = maximum; Min = minimum; $\mathrm{n}=$ number of subjects; $\mathrm{SD}=$ standard deviation

Notes: For the hair follicle and buccal swab collection types, the mean FMR1 mRNA Delta Cq value across all samples for each subject is summarized.

Buccal and hair follicle FMR1 mRNA were quantified by qRT-PCR and blood FMR1 was measured by PrimeFlow ${ }^{\mathrm{TM}}$. The 2 measurements cannot be directly compared across sample types. Within sample type comparisons between healthy participants and FXS participants are appropriate.

For the blood collection type, two Relative FMR1 values collected using PrimeFlow ${ }^{\mathrm{TM}}$ technology were reported for Subjects 101-002, 101-005, and 101007; only the smallest value for each subject is summarized.

For FMR1 PrimeFlow ${ }^{\mathrm{TM}}$ values are reported as FMRP/IC (dapB) Median Fluorescent Intensity (MFI).

In FXS participants, both the hair follicle and blood lymphocyte cells showed null (0.0) values. One null value in one hair follicle was seen for FMRP in one healthy participant. The point of failure for any lysate used for MSD was a total protein concentration below the lower limit of quantification (LLOQ) of the total protein assay. A smaller number of FXS subjects did not achieve hair follicle FMRP positivity on Visit 2 due to MSD results being below LLOQ.

Both blood lymphocyte and hair follicle FMRP levels measured by MSD were lower by a factor of 5 to 6 in FXS participants compared to healthy participants. In all subjects, FMRP could not be detected in buccal mucosa sampled for FMRP determination in this study. Of the 2 subjects who were negative for FMRP using blood samples, one of them also was negative for FMRP in the hair follicle sample.

FMRP was quantified in hair follicles using only the MSD ELISA. FMRP was quantified in PBMCs using two methods. An absolute quantity in fmol/ $\mu$ g total protein was determined using the MSD ELISA; and a relative quantification in a ratio of FMRP Mean Fluorescence Intensity to Isotype Control Mean Fluorescence Intensity using the multiparameter, flow cytometric assay PrimeFlow ${ }^{\mathrm{TM}}$. Regardless of the method to measure FMRP in PBMCs, the amount of FMRP was significantly lower in the FXS participants versus the healthy participants (Table 3).

Per follicle FMRP levels detected using MSD showed clustering by FXS diagnosis with higher FMRP levels seen in healthy controls compared to subjects with the full mutation or mosaic subjects (Figure 1). Inter-follicle variability was lowest in mosaic subjects with the majority of follicles showing FMRP levels less than $25 \mathrm{fmol} / \mu \mathrm{g}$ protein.

Mean FMRP in hair follicles versus PBMCs was correlated to mutation status with healthy controls showing high levels of FMRP and mosaic subjects showing low levels of FMRP when assessed using MSD (Figure 2). Full mutation subjects showed intermediate FMRP levels compared to healthy controls and mosaic subjects; 4 full mutation subjects, however, showed low FMRP levels with the hair follicle method of which 2 subjects showed intermediate levels and 2 subjects showed corroborating low levels with the PBMCs (overall, $n=6$ ). Correlations using PBMCs between the PrimeFlow ${ }^{\text {TM }}$ and MSD ELISA methods showed a similar pattern of high levels of FMRP in healthy control (male/female subjects), intermediate levels of FMRP in full mutation female subjects, and low levels in full mutation male subjects and in mosaic (male/female) subjects, respectively.

Hair follicle mean FMR1 levels were lower in FXS participants compared to healthy participants (Table 5). The mean hair follicle FMR1 level using qRT-PCR in FXS participants was $0.055 \pm 0.10$ Delta Cq and $0.127 \pm 0.11$ Delta Cq compared to $0.165 \pm 0.15$ Delta Cq and $0.184 \pm 0.14$ Delta Cq for healthy participants at Visit 1 and Visit 2, respectively. Mean FMR1 in blood lymphocytes assessed using PrimeFlow ${ }^{\mathrm{TM}}$ showed less variation in relative terms at $1.026 \pm 0.08$ FMR1/dapB MFI and $0.905 \pm 0.15 \mathrm{FMR} 1 /$ dapB MFI in FXS participants and 1.197 $\pm 0.08 \mathrm{FMR} 1 /$ dapB MFI and $1.164 \pm 0.06 \mathrm{FMR} 1 /$ dapB MFI in healthy participants at Visit 1 and Visit 2, respectively, compared to hair follicle FMR1 levels using qRT-PCR. FMR1 was not quantified in PBMCs using qRT-PCR. Mean FMR1 assessed by qRT-PCR using the buccal swab showed a mean level of $0.354 \pm 0.38$ Delta Cq and $0.443 \pm 0.54$ Delta Cq in FXS participants compared to 
$1.108 \pm 0.85$ Delta Cq and $0.772 \pm 0.34$ Delta Cq for healthy participants at Visit 1 and Visit 2, respectively. Higher FMR 1 levels were generally seen in healthy controls and mosaic subjects although there were a number of healthy control and mosaic follicles with intermediate and low FMR1 levels The majority of full mutation subjects demonstrated low FMR1 levels.

Table 5

FMR1 Levels and Changes from Baseline by Collection Type and Visit

\begin{tabular}{|c|c|c|c|c|c|c|c|c|c|c|c|c|c|}
\hline \multirow[b]{2}{*}{ Collection Type } & \multirow[b]{2}{*}{ Visit } & \multicolumn{6}{|c|}{ Reported Value } & \multicolumn{6}{|c|}{ Change from Baseline Value* } \\
\hline & & $\mathrm{n}$ & Mean & SD & Median & Min & Max & $\mathrm{n}$ & Mean & SD & Median & Min & Max \\
\hline \multicolumn{14}{|c|}{ FXS Participant (N=9) } \\
\hline \multirow[t]{2}{*}{ Buccal Swab } & Visit 1 & 8 & 0.354 & 0.3782 & 0.363 & 0.000 & 1.064 & & & & & & \\
\hline & Visit 2 & 7 & 0.443 & 0.5438 & 0.235 & 0.000 & 1.471 & 5 & 0.138 & 0.7450 & 0.056 & -0.840 & 1.108 \\
\hline \multirow[t]{2}{*}{ Hair Follicle } & Visit 1 & 8 & 0.055 & 0.0952 & 0.030 & 0.000 & 0.288 & & & & & & \\
\hline & Visit 2 & 9 & 0.127 & 0.1055 & 0.078 & 0.000 & 0.228 & 8 & 0.082 & 0.1665 & 0.111 & -0.247 & 0.271 \\
\hline \multirow[t]{2}{*}{ Blood } & Visit 1 & 7 & 1.026 & 0.0750 & 0.984 & 0.965 & 1.148 & & & & & & \\
\hline & Visit 2 & 3 & 0.905 & 0.1476 & 0.830 & 0.811 & 1.075 & & & & & & \\
\hline \multicolumn{14}{|c|}{ Healthy Participant $(\mathrm{N}=5)$} \\
\hline \multirow[t]{2}{*}{ Buccal Swab } & Visit 1 & 5 & 1.108 & 0.8453 & 0.784 & 0.523 & 2.341 & & & & & & \\
\hline & Visit 2 & 5 & 0.772 & 0.3437 & 0.733 & 0.394 & 1.228 & 4 & -0.336 & 0.8542 & -0.004 & -1.595 & 0.258 \\
\hline \multirow[t]{2}{*}{ Hair Follicle } & Visit 1 & 5 & 0.165 & 0.1504 & 0.195 & 0.000 & 0.370 & & & & & & \\
\hline & Visit 2 & 5 & 0.184 & 0.1407 & 0.158 & 0.077 & 0.423 & 5 & 0.019 & 0.1874 & 0.077 & -0.212 & 0.228 \\
\hline \multirow[t]{2}{*}{ Blood } & Visit 1 & 4 & 1.197 & 0.0842 & 1.181 & 1.124 & 1.303 & & & & & & \\
\hline & Visit 2 & 4 & 1.164 & 0.0604 & 1.152 & 1.108 & 1.245 & 1 & -0.119 & & -0.119 & -0.119 & -0.119 \\
\hline \multicolumn{14}{|c|}{ Abbreviations: Max = maximum; Min = minimum; $\mathrm{n}$ = number of subjects; $\mathrm{SD}=$ standard deviation } \\
\hline \multicolumn{14}{|c|}{ *Baseline is defined as value collected during Visit 1 . Change from baseline values are calculated as the assessment value minus the baseline value. } \\
\hline \multicolumn{14}{|c|}{ Notes: For the hair follicle and buccal swab collection types, the mean FMR1 mRNA Delta Cq value across all samples for each subject is summarized. } \\
\hline
\end{tabular}

In hair follicles, mean FMRP by MSD ELISA did not correlate with mean FMR1 by qRT-PCR in healthy controls compared to mosaic and full mutation subjects and full mutation subjects also showed no correlation compared to mosaic subjects. All mosaic subjects $(n=4)$ showed low levels of FMRP expression. In PBMCs, mean FMRP positively correlated with mean FMR1 in both healthy controls and full mutation participants. PrimeFlow ${ }^{\text {TM }}$ (a single cell method) was used to measure both FMRP and FMR1 simultaneously in PBMCs. By PrimeFlow ${ }^{\text {TM }}$, PBMCs from mosaic participants gave 2 distinct FMR1 populations; therefore, results from mosaic participants were not included.

A positive Pearson's r correlation was seen between FMR1 levels and the OWLS-II LC and OE subscale scores in FXS subjects. The highest correlation between FMR 1 levels and OWLS-II LC and OE scores was seen for the FXS blood samples ( 0.780 and 0.774 , respectively), followed by samples collected by buccal swab ( 0.380 and 0.368 , respectively) and hair follicle plucking ( 0.017 and 0.021 , respectively) (see Table 6$)$. This data suggests that measurements in blood better reflect the clinical phenotype than measurements in hair follicles or buccal swabs. In comparison to FXS subjects, OWLS-II subscale scores for healthy subjects did not show the same level of positive correlation with FMR1 levels. For the GARS-3, positive Pearson's r correlations were seen between FMR1 levels and the social communication and cognitive style subscales in healthy subjects; no correlation was seen in FXS subjects between FMR1 levels and GARS-3 subscale scores. 
Table 6

Relationship Between FMR1 Levels and OWLS-II and GARS-3 Scores at Visit 1 - Pearson Correlation Coefficients (Enrolled Analysis Set)

\begin{tabular}{|c|c|c|c|c|c|c|c|c|c|}
\hline & & OWLS-II Subsca & & GARS-3 Subscale & & & & & \\
\hline \multirow[t]{2}{*}{ Group } & \multirow[t]{2}{*}{$\begin{array}{l}\text { Collection } \\
\text { Type }\end{array}$} & \multirow[t]{2}{*}{$\begin{array}{l}\text { Listening } \\
\text { Comprehension }\end{array}$} & \multirow[t]{2}{*}{$\begin{array}{l}\text { Oral } \\
\text { Expression }\end{array}$} & & Social & Social & Emotional & Cognitive & Maladapt \\
\hline & & & & Behaviors & Interaction & Communication & Responses & Style & Speech \\
\hline \multirow{3}{*}{$\begin{array}{l}\text { FXS } \\
\text { Participant } \\
(\mathrm{N}=9)\end{array}$} & $\begin{array}{l}\text { Buccal } \\
\text { Swab }\end{array}$ & 0.380 & 0.368 & -0.400 & -0.188 & -0.212 & -0.419 & -0.272 & -0.430 \\
\hline & $\begin{array}{l}\text { Hair } \\
\text { Follicle }\end{array}$ & -0.017 & 0.021 & 0.072 & -0.170 & 0.036 & -0.071 & 0.439 & 0.310 \\
\hline & Blood & 0.780 & 0.774 & -0.586 & -0.427 & -0.336 & -0.337 & 0.085 & -0.521 \\
\hline \multirow{3}{*}{$\begin{array}{l}\text { Healthy } \\
\text { Participant } \\
(\mathrm{N}=5)\end{array}$} & $\begin{array}{l}\text { Buccal } \\
\text { Swab }\end{array}$ & -0.353 & -0.047 & $\mathrm{Nc}$ & $\mathrm{nc}$ & 0.598 & $\mathrm{nc}$ & 0.709 & $\mathrm{nc}$ \\
\hline & $\begin{array}{l}\text { Hair } \\
\text { Follicle }\end{array}$ & -0.001 & -0.557 & $\mathrm{Nc}$ & $\mathrm{nc}$ & -0.263 & 0.344 & 0.162 & nc \\
\hline & Blood & 0.289 & 0.316 & $\mathrm{Nc}$ & $\mathrm{nc}$ & 0.369 & -0.230 & 0.302 & $\mathrm{nc}$ \\
\hline
\end{tabular}

Abbreviations: GARS-3 = Gilliam Autism Rating Scale 3rd Edition; $\mathrm{nc}=$ not calculable due to failure of a majority of samples to yield adequate sample to be analyzed; OWLS-II = Oral and Written Language Scale 2nd Edition.

Notes: For the hair follicle and buccal swab collection types, the mean FMR1 mRNA Delta Cq value across all samples is correlated with each OWLS-II score $\mathrm{C}$ each GARS-3 score.

For the blood collection type, two Relative FMR1 values were reported for Subjects 101-002, 101-005, and 101-007; only the smallest value for each subject is used in correlations.

FMRP levels also showed a high level of positive correlation with the OWLS-II LC and OE subscale scores in FXS subjects, with relatively higher correlation seen for blood samples compared to hair follicle samples (see Table 7). In blood, the Pearson's r correlation was 0.802 and 0.817 between FMRP levels and the OWLS-II LC and OE subscale, respectively. For the GARS-3, a positive correlation was seen between FMR1 levels and the social communication and cognitive style subscales with blood samples in healthy subjects while no corresponding correlation was seen for FXS subjects.

Table 7

Relationship Between FMRP Levels and OWLS-II Scores and GARS-3 Scores at Visit 1 - Pearson Correlation Coefficients (Enrolled Analysis Set)

\begin{tabular}{|c|c|c|c|c|c|c|c|c|c|}
\hline & & OWLS-II Subscal & & GARS-3 Subscale & & & & & \\
\hline \multirow[t]{2}{*}{ Group } & \multirow{2}{*}{$\begin{array}{l}\text { Collection } \\
\text { Type }\end{array}$} & \multirow{2}{*}{$\begin{array}{l}\text { Listening } \\
\text { Comprehension }\end{array}$} & \multirow{2}{*}{$\begin{array}{l}\text { Oral } \\
\text { Expression }\end{array}$} & Restricted/Repetitive & Social & Social & Emotional & Cognitive & Maladapti \\
\hline & & & & Behaviors & Interaction & Communication & Responses & Style & Speech \\
\hline \multirow{2}{*}{$\begin{array}{l}\text { FXS } \\
\text { Participant } \\
(\mathrm{N}=9)\end{array}$} & $\begin{array}{l}\text { Hair } \\
\text { Follicle }\end{array}$ & 0.267 & 0.507 & -0.506 & 0.335 & 0.073 & -0.253 & -0.153 & -0.491 \\
\hline & Blood & 0.802 & 0.817 & -0.586 & -0.201 & -0.175 & -0.154 & -0.115 & -0.631 \\
\hline \multirow{2}{*}{$\begin{array}{l}\text { Healthy } \\
\text { Participant } \\
(\mathrm{N}=5)\end{array}$} & $\begin{array}{l}\text { Hair } \\
\text { Follicle }\end{array}$ & 0.195 & 0.347 & $\mathrm{nc}$ & $\mathrm{nc}$ & 0.031 & -0.243 & -0.192 & $\mathrm{nc}$ \\
\hline & Blood & -0.030 & -0.567 & nc & $\mathrm{nc}$ & 0.278 & -0.066 & 0.695 & $\mathrm{nc}$ \\
\hline
\end{tabular}

Sources: Table 14.2.9, Table 14.2.10, Listing 16.2.4.5, Listing 16.2.4.6, Listing 16.2.6.6.

Abbreviations: GARS-3 = Gilliam Autism Rating Scale 3rd Edition; nc = not calculable due to failure of a majority of samples to yield adequate sample to be analyzed; OWLS-II = Oral and Written Language Scale 2nd Edition

Notes: For the hair follicle collection type, the mean fmol FMRP/ug protein value across all samples is correlated with each OWLS-II score or each GARS-3 score.

For the buccal swab collection type, the fmol FMRP/ug protein value is not summarized as the value was 'Below LLOQ' for all samples. 


\section{Discussion:}

This was a single center, prospective, non-drug pilot biomarker feasibility study. We assessed repeated collection of hair follicles by plucking in individuals with FXS and those who were typically developing for the quantitative measurement of FMRP and FMR1 mRNA. Results were compared to blood and buccal swabs and collected at two different locations, in their home and in the clinic. The primary objective of this study was to determine whether repeated collection of scalp hair follicles by plucking is feasible in individuals with FXS. Secondary objectives included comparison of the completeness of data in office visits versus home visits, comparisons of the positivity and levels of FMRP and FMR1 in hair follicles by collection type and collection location, and assessment of associations between FMRP and FMR1 obtained by above methods with measures of clinical severity.

Mostly young males and female subjects were enrolled into the study with all FXS subjects confirmed with the full mutation, almost half of which were mosaic positive. For full FXS subjects the mean OWLS-II LC score was $82 \pm 16.7$ (Range 54 to 108) and OWLS-II OE score was $73 \pm 16.8$ (Range 48 to 95 ). Scores below 70 are considered deficient (OWLS-II: average $=85-115$; below average $=70-84$; deficient $=$ less than 70).

This study determined that the repeated collection of scalp follicles by plucking was a feasible method of collection in children with FXS. For the FXS group, a mean of $4.7 \pm 1.00$ hair follicles per subject were collected at the office visit ( $n=9$ ) versus a mean of $6.1 \pm 2.23$ hair follicles per subject at the home visit ( $n=8$ ). This was similar to the collection in healthy participants with a mean of $5.2 \pm 2.17$ hair follicles collected per subject at the office visit ( $n=5$ ) compared to a mean of $4.8 \pm 1.79$ hair follicles per subject at the home visit $(n=5)$.

Using the newly developed method of MSD ELISA, similar mean percentages of FMRP positive hair follicles obtained using plucking were seen from FXS and healthy participants (FXS participants - office: $81.3 \%$, home: 59.3\%; healthy participants - office: 64.1\%, home: 70.0\%). Absolute levels of FMRP and FMR1 mRNA were substantially higher in healthy participants compared to full mutation and mosaic FXS participants, and lowest in the FXS boys. Importantly, measurement of FMRP and FMR1 levels did not show any notable variation by collection location at home versus the office across the various tested sample collection methodologies of hair follicle, blood sample, and buccal swab.

In hair follicles, we observed no relationship between FMRP measured by MSD and FMR1 measured by qRT-PCR. However, in PBMCs using the novel PrimeFlow ${ }^{\mathrm{TM}}$ assay, we did observe a strong relationship between the relative amounts of FMRP and FMR1. Further studies may be warranted to determine if the lack of relationship in hair follicles for FMRP and FMR1 is due to the use of divergent techniques or if it is a property of hair follicles or the collection methods. In general, there was little variation in FMRP and FMR1 levels between successive visits indicating a favorable repeatability profile for hair follicle sampling, an important characteristic for potential use of one or more of these biomarkers in the context of future therapeutic clinical trials that seek to reactivate FMR1/FMRP. Notably, hair follicle values demonstrated that if a sufficient number of samples are obtained from FXS participants, FMRP is not only detectable but also quantifiable in full mutation participants. Use of blood lymphocytes allow for only one sampling per visit. The sampling superiority of hair follicles versus blood lymphocytes may allow for the quantification of FMRP by MSD in full mutation individuals and allows a determination of percent positive follicles.

Strong positive correlations were seen between FMRP and FMR1 levels in blood and the OWLS-II LC and OE subscale scores in FXS subjects, and of greater magnitude than for hair follicles and buccal swabs. This confirms our previous recent finding in a separate population of children with FXS (20). For blood samples, the pearson's $r$ correlation was highly significant at 0.802 and 0.817 between FMRP levels and the OWLS-II LC and OE subscale, respectively. Thus, the study provides strong support for the use of blood samples to measure longitudinal changes in FMRP/FMR1 in future therapeutic clinical trials seeking to treat the root cause of FXS. The strong correlations of blood with clinical outcome assessments of communication such as OWLS-II support the clinical significance of this biomarker. Moreover, these findings are similar to those demonstrated in Roth et al., 2021, in which a positive association between IQ and FMRP expression in PBMCs by either, MSD or PrimeFlow ${ }^{T M}$ was noted. However, several studies have demonstrated that venipuncture in children with ASD and other neurodevelopmental disorders can lead to significant distress, behavioral disturbance, noncompliance with study procedures, and withdrawal from the study $(24,25)$. These findings were corroborated in the current study as well where we noted that blood samples were only obtained from 6 out of 10 FXS participants. Future work may improve the feasibility of repeated collection of blood in children with FXS.

\section{Conclusion:}

In summary, our data demonstrated that repeated sampling of hair follicles in individuals with FXS, in both, home and office settings, is a feasible method for measurement of FMRP and FMR1 levels. While FMRP and FMR1 obtained from hair follicles did not demonstrate strong relationships with language and behavioral measures, little variation in their levels during successive visits was noted, which is an important feature in biomarker development to plan future studies. Furthermore, the current study provides support for the use of repeated hair follicle sampling in future clinical therapeutic studies as an effective biomarker of FMRP and FMR1 levels and highlights the need to improve the feasibility of repeated collection of blood

\section{Declarations}

\section{Availability of Data and Materials:}

The datasets generated during and/or analyzed during the current study are available from the corresponding author on reasonable request.

\section{Acknowledgements:}

The authors would like to express their gratitude to all the children and parents who participated in this study.

Funding:

Page $12 / 15$ 
This research was supported by Fulcrum Therapeutics, Cambridge, MA.

\section{Conflicts of Interest:}

M.R. is a full-time employee of Fulcrum Therapeutics and owns equity shares in Fulcrum Therapeutics. L.R. and D.C. were full-time employees of Fulcrum Therapeutics during the experimental work and writing of the manuscript. They are no longer with Fulcrum Therapeutics, but they own equity in Fulcrum Therapeutics. D.C. is currently a full-time employee of X4 Pharmaceuticals, which is not related to this work. J.A.F. receives research support from Healix. D.M.C. consults with Abvie Inc. The other authors do not have any conflicts of interest to declare.

\section{Ethics approval and consent to participate:}

This study was approved by the Institutional Review Board of the University of Massachusetts Chan Medical School (\#H00013963). Written informed consent was obtained from a parent or legal guardian prior to the study.

\section{Contributions:}

IJ, JAF, MR, DMC, LR, DC and TM were major contributors in writing the manuscript. JAF, MR, LR and DC were involved in the conceptualization of the study. MR conducted the data analysis. AF and LV were involved with writing the protocol, recruitment, assessment, and review of data.

\section{Consent for Publication:}

N/A

\section{References}

1. Hagerman RJ, Staley LW, O'Conner R, Lugenbeel K, Nelson D, McLean SD, et al. Learning-disabled males with a fragile X CGG expansion in the upper premutation size range. Pediatrics. 1996;97(1):122-6. https://doi.org/10.1542/peds.97.1.122

2. Kaufmann WE, Abrams MT, Chen W, Reiss AL. Genotype, molecular phenotype, and cognitive phenotype: correlations in fragile X syndrome. American journal of medical genetics. 1999;83(4):286-95. https://doi.org/10.1002/(SICI)1096-8628(19990402)83:4<286::AID-AJMG10>3.0.CO;2-H

3. HessI D, Dyer-Friedman J, Glaser B, Wisbeck J, Barajas RG, Taylor A, et al. The influence of environmental and genetic factors on behavior problems and autistic symptoms in boys and girls with fragile X syndrome. Pediatrics. 2001;108(5):e88-e. https://doi.org/10.1542/peds.108.5.e88

4. Hunter J, Rivero-Arias O, Angelov A, Kim E, Fotheringham I, Leal J. Epidemiology of fragile X syndrome: A systematic review and meta-analysis. American Journal of Medical Genetics Part A. 2014;164(7):1648-58. https://doi.org/10.1002/ajmg.a.36511

5. Klaiman C, Quintin E-M, Jo B, Lightbody AA, Hazlett HC, Piven J, et al. Longitudinal profiles of adaptive behavior in fragile X syndrome. Pediatrics. 2014;134(2):315-24. https://doi.org/10.1542/peds.2013-3990

6. LaFauci G, Adayev T, Kascsak R, Brown WT. Detection and quantification of the fragile X mental retardation protein 1 (FMRP). Genes. $2016 ; 7(12): 121$. https://doi.org/10.3390/genes7120121

7. Pretto D, Yrigollen CM, Tang H-T, Williamson J, Espinal G, Iwahashi CK, et al. Clinical and molecular implications of mosaicism in FMR1 full mutations. Frontiers in genetics. 2014;5:318. https://doi.org/10.3389/fgene.2014.00318

8. Loesch DZ, Huggins RM, Bui QM, Epstein JL, Taylor AK, Hagerman RJ. Effect of the deficits of fragile X mental retardation protein on cognitive status of fragile $X$ males and females assessed by robust pedigree analysis. Journal of Developmental \& Behavioral Pediatrics. 2002;23(6):416-23.

9. Loesch D, Huggins R, Bui Q, Taylor A, Pratt C, Epstein J, et al. Effect of fragile X status categories and FMRP deficits on cognitive profiles estimated by robust pedigree analysis. American Journal of Medical Genetics Part A. 2003;122(1):13-23. https://doi.org/10.1002/ajmg.a.20214

10. Saldarriaga W, Tassone F, González-Teshima LY, Forero-Forero JV, Ayala-Zapata S, Hagerman R. Fragile X syndrome. Colombia medica. 2014;45(4):190-8.

11. Jalnapurkar I, Cochran DM, Frazier JA. New therapeutic options for fragile X syndrome. Current treatment options in neurology. 2019;21(3):1-14. DOI: 10.1007/s11940-019-0551-8

12. Harris SW, HessI D, Goodlin-Jones B, Ferranti J, Bacalman S, Barbato I, et al. Autism profiles of males with fragile X syndrome. American Journal on Mental Retardation. 2008;113(6):427-38. https://doi.org/10.1352/2008.113:427-438

13. Schaefer TL, Davenport MH, Erickson CA. Emerging pharmacologic treatment options for fragile X syndrome. The application of clinical genetics. 2015;8:75. DOI: 10.2147/TACG.S35673

14. Ligsay A, Hagerman RJ. Review of targeted treatments in fragile X syndrome. Intractable \& rare diseases research. 2016. DOI: 10.5582/irdr.2016.01045

15. Pan F, Aldridge GM, Greenough WT, Gan W-B. Dendritic spine instability and insensitivity to modulation by sensory experience in a mouse model of fragile X syndrome. Proceedings of the National Academy of Sciences. 2010;107(41):17768-73. https://doi.org/10.1073/pnas.1012496107

16. Tabolacci E, Palumbo F, Nobile V, Neri G. Transcriptional reactivation of the FMR1 gene. a possible approach to the treatment of the fragile X syndrome. Genes. 2016;7(8):49. https://doi.org/10.3390/genes7080049

17. Chiurazzi P, Pomponi MG, Willemsen R, Oostra BA, Neri G. In vitro reactivation of the FMR1 gene involved in fragile X syndrome. Human molecular genetics. 1998;7(1):109-13. https://doi.org/10.1093/hmg/7.1.109

18. Biacsi R, Kumari D, Usdin K. SIRT1 inhibition alleviates gene silencing in Fragile X mental retardation syndrome. PLoS genetics. 2008;4(3):e1000017. https://doi.org/10.1371/journal.pgen.1000017 
19. Willemsen R, Anar B, Otero YDD, de Vries BB, Hilhorst-Hofstee Y, Smits A, et al. Noninvasive test for fragile X syndrome, using hair root analysis. The American Journal of Human Genetics. 1999;65(1):98-103. https://doi.org/10.1086/302462

20. Roth M, Ronco L, Cadavid D, Durbin-Johnson B, Hagerman RJ, Tassone F. FMRP Levels in Human Peripheral Blood Leukocytes Correlates with Intellectual Disability. Diagnostics. 2021;11(10):1780. https://doi.org/10.3390/diagnostics11101780

21. Budimirovic DB, Schlageter A, Filipovic-Sadic S, Protic DD, Bram E, Mahone EM, et al. A genotype-phenotype study of high-resolution FMR1 nucleic acid and protein analyses in fragile X patients with neurobehavioral assessments. Brain sciences. 2020;10(10):694. https://doi.org/10.3390/brainsci10100694

22. Carrow-Woolfolk E. Oral and written language scales: Circle Pines, MN: American Guidance Service; 1995.

23. Gilliam JE. GARS: Gilliam autism rating scale: Pro-ed; 1995.

24. Duff A. Incorporating psychological approaches into routine paediatric venepuncture. Archives of Disease in Childhood. 2003;88(10):931-7. http://dx.doi.org/10.1136/adc.88.10.931

25. Davit CJ, Hundley RJ, Bacic JD, Hanson EM. A pilot study to improve venipuncture compliance in children and adolescents with autism spectrum disorders. Journal of developmental and behavioral pediatrics: JDBP. 2011;32(7):521. DOI: 10.1097/DBP.0b013e3182245b09

\section{Figures}

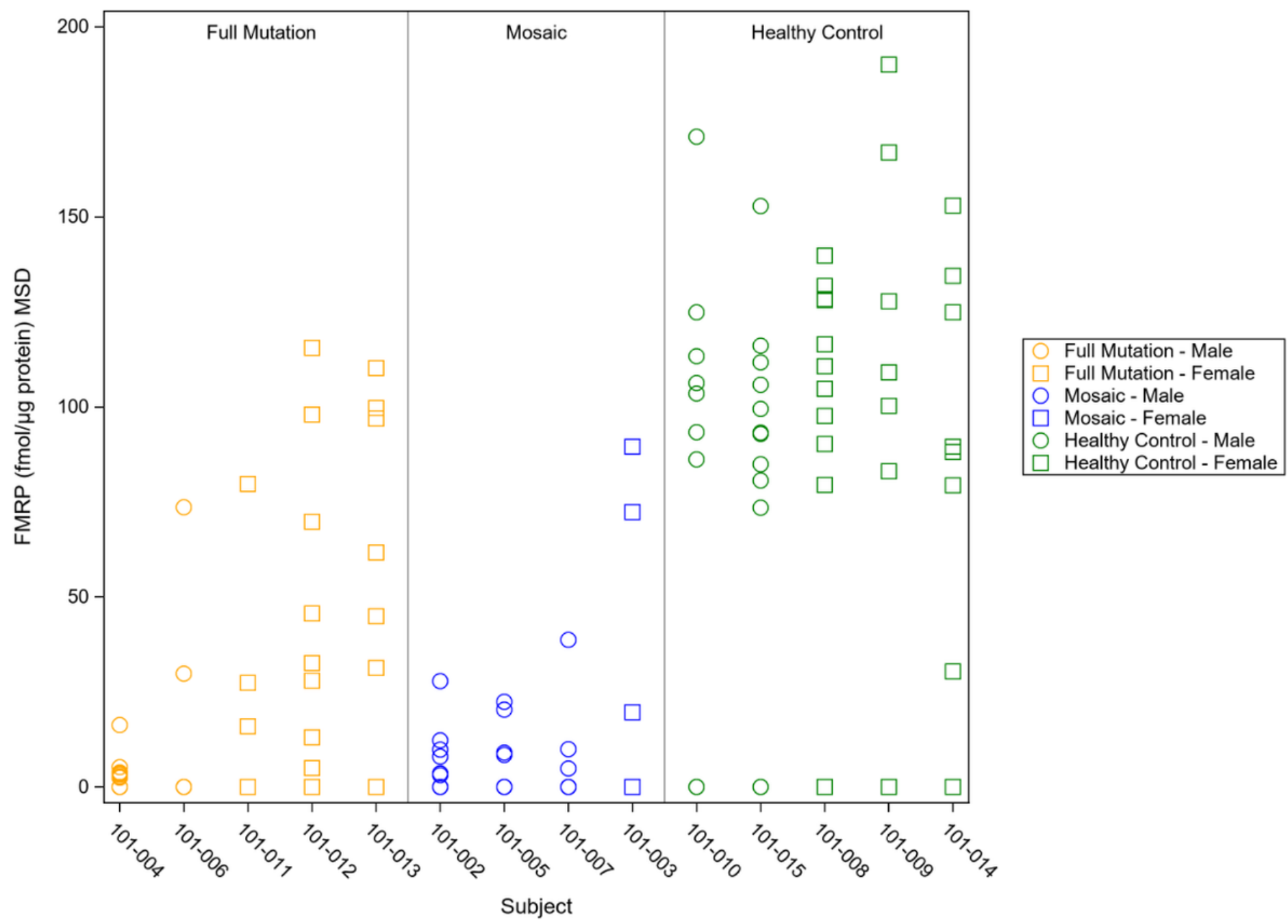

Figure 1

FMRP Level per Sampled Hair Follicle by Subject Clustered by Group and Sex 


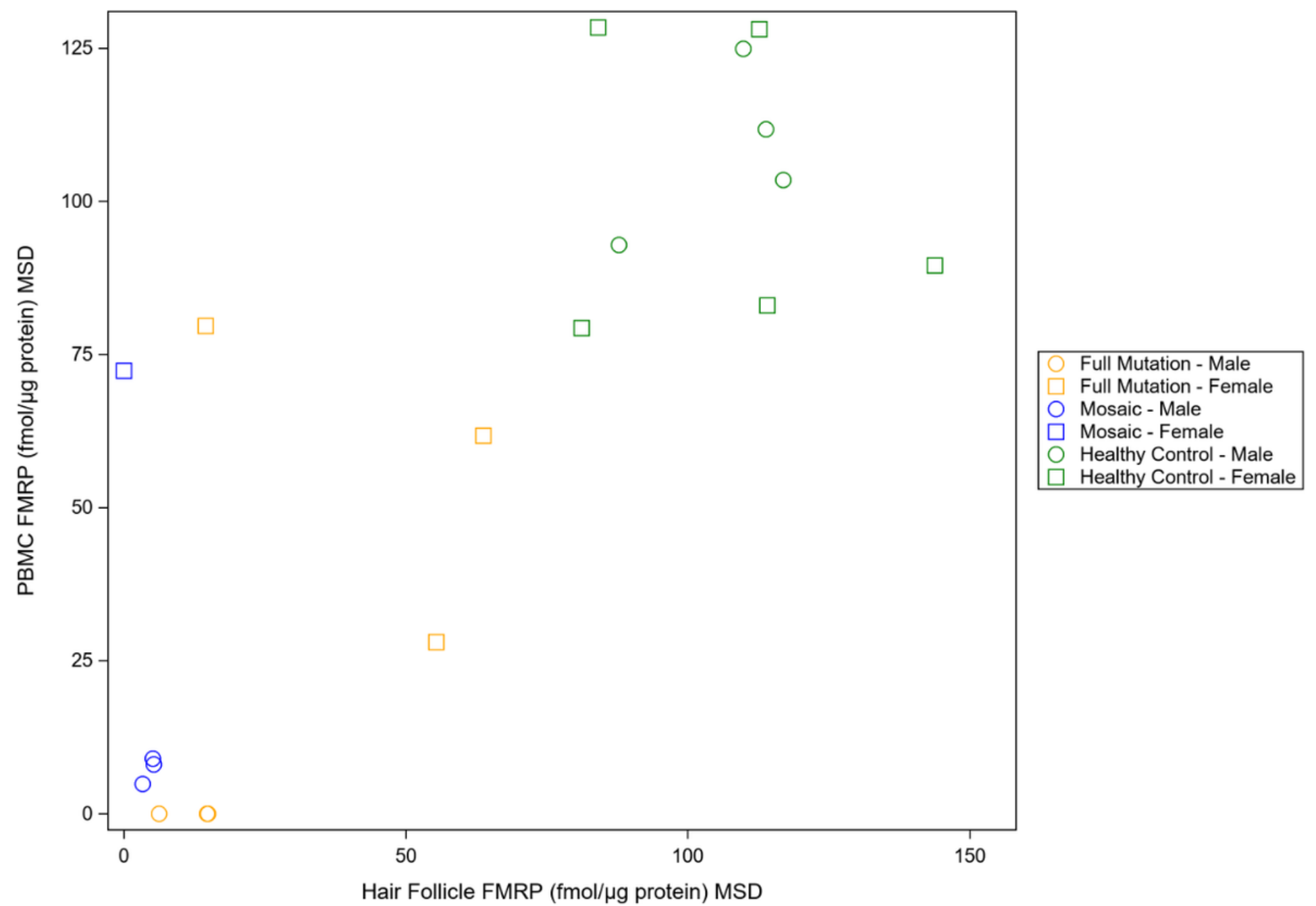

Figure 2

Scatterplot of Mean FMRP Level Across Sampled Hair Follicles and PBMC FMRP Level Clustered by Group and Sex 